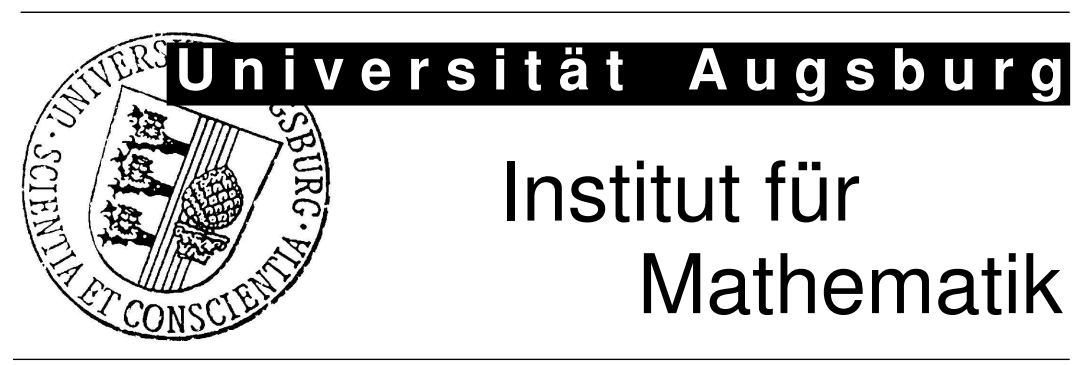

Harbir Antil, Ronald H.W. Hoppe, Christopher Linsenmann Adaptive Multilevel Interior-Point Methods in PDE Constrained Optimization 


\section{Impressum:}

Herausgeber:

Institut für Mathematik

Universität Augsburg

86135 Augsburg

http://www . math. uni-augsburg.de/forschung/preprint/

ViSdP:

Ronald H.W. Hoppe

Institut für Mathematik

Universität Augsburg

86135 Augsburg

Preprint: Sämtliche Rechte verbleiben den Autoren (C) 2008 


\title{
Adaptive Multilevel Interior-Point Methods in PDE Constrained Optimization
}

\author{
Harbir Antil ${ }^{1}$, Ronald H.W. Hoppe ${ }^{1,2}$, and Christopher Linsenmann ${ }^{1,2}$ \\ 1 University of Houston, Department of Mathematics \\ (http://www.math.uh.edu/ rohop/) \\ 2 University of Augsburg, Institute for Mathematics \\ (http://scicomp.math.uni-augsburg.de)
}

Summary. We are concerned with structural optimization problems where the state variables are supposed to satisfy a PDE or a system of PDEs and the design variables are subject to inequality constraints. Within a primal-dual setting, we suggest an all-at-once approach based on interior-point methods. Coupling the inequality constraints by logarithmic barrier functions involving a barrier parameter and the PDE by Lagrange multipliers, the KKT conditions for the resulting saddle point problem represent a parameter dependent nonlinear system. The efficient numerical solution relies on multilevel path-following predictor-corrector techniques with an adaptive choice of the continuation parameter where the discretization is taken care of by finite elements with respect to nested hierarchies of simplicial triangulations of the computational domain. In particular, the predictor is a nested iteration type tangent continuation, whereas the corrector is a multilevel inexact Newton method featuring transforming null space iterations. As an application in life sciences, we consider the optimal shape design of capillary barriers in microfluidic biochips.

\section{Introduction}

The optimization of structures and systems has a long history that can be traced back to the work of Bernoulli, Euler, Lagrange, and Saint-Venant. It became its own discipline during the second half of the last century when the rapid progress in electronic data processing required the development and implementation of highly efficient and robust algorithmic optimization tools. Nowadays, shape optimization is an indispensable tool for many design issues in aero- and fluid dynamics, electromagnetics, and structural mechanics.

The spectrum of analytical and numerical methods is well documented by numerous monographs on the subject that have been published during the past twenty-five years (cf., e.g., Allaire (2002); Bendsøe (1995); Delfour and Zolesio (2001); Haslinger and Neittaanmäki (1988); Haslinger and Mäkinen (2004); Mohammadi and Pironneau (2001); Pironneau (1984); Sokolowski 
and Zolesio (1992)).

In this paper, we will focus on an all-at-once approach by means of primal-dual interior-point methods. Using classical barrier functions, this results in a parameter dependent nonlinear system which is solved by a multilevel predictorcorrector continuation strategy with an adaptive choice of the continuation steplength along the central path. The predictor relies on a nested iteration type continuation, whereas the corrector features an inexact Newton method involving transforming null space iterations as inner iterations. As a multiscale multiphysics application, we consider the optimal design of capillary barriers in surface acoustic wave driven microfluidic biochips used for hybridization and sequencing in genomics.

\section{Optimal design of processes and systems}

A typical shape optimization problem associated with a time-independent PDE or a system thereof as the underlying state equation amounts to the minimization of a shape functional $J$ over bounded domains $\Omega$ in Euclidean space $\mathbb{R}^{d}$. The state function $u$ is assumed to satisfy a boundary value problem as described by means of a partial differential operator $L$, and there may be further equality and/or inequality constraints on the domain.

$$
\inf _{\Omega} J(u, \Omega) \quad, \quad J(u, \Omega):=\int_{\Omega} j(x, u(x)) d x,
$$

$$
\text { subject to } L u=f \quad \text { in } \Omega, \quad u=g \text { on } \Gamma \quad, \quad h(\Omega) \geq 0 .
$$

The inherent difficulty that the minimization is over a certain class of domains instead of a set of functions in an appropriate function space can be circumvented by the so-called shape calculus as developed by Céa, Delfour, Zolésio and others (cf., e.g., Delfour and Zolesio (2001)). The necessary optimality conditions can be stated by means of the shape gradient

$$
\nabla J(\Omega)[V]=\lim _{t \rightarrow 0+} \frac{J\left(\Omega_{t}(V)\right)-J(\Omega)}{t}=\langle\nabla J(\Omega),
$$

where $V \in X:=C^{2, \alpha}\left(\hat{\Omega} ; \mathbb{R}^{d}\right)$. The shape gradient is a distributional derivative that can be defined by the velocity method in terms of velocity fields $V$ describing transformation of domains. The shape gradient admits a boundary integral representation

$$
\langle\nabla J(\Omega), V\rangle=\int_{\Gamma}\langle V, \nu\rangle\left\{j(x, g)+\frac{\partial p}{\partial \nu} \frac{\partial(g-u)}{\partial \nu}\right\} d s,
$$

involving the adjoint state $p$ that satisfies the adjoint state equation $L^{*} p=$ $\partial j / \partial u(\cdot, u)$. Sufficient optimality conditions invoke the shape Hessian which 
can also be given a boundary integral representation admitting an interpretation as a pseudo differential operator of order 1 (cf., e.g., Eppler and Harbrecht (2006)). The analytical investigation of shape Hessians and the development and implementation of numerical tools based thereon is subject to intensive ongoing research. The numerical methods developed so far require some smoothness of the domain and suffer from a lack of stability otherwise. Since interior-point methods essentially rely on second order information, in the sequel we will use a more classical approach based on a parametrization of the domain by a finite number of design variables. The boundary $\Gamma$ is represented by a composite Bézier curve using a certain number of Bézier control points $\alpha \in \mathbb{R}^{m}, m \in \mathbb{N}$, which serve as design variables. The equality and/or inequality constraints are expressed by means of the design variables. For the finite element approximation of (1a)-(1b) we choose $\hat{\alpha}$ as a reference design and refer to $\hat{\Omega}:=\Omega(\hat{\alpha})$ as the associated reference domain. Then, the actual domain $\Omega(\alpha)$ can be obtained from the reference domain $\hat{\Omega}$ by means of a mapping $\Omega(\alpha)=\Phi(\hat{\Omega} ; \alpha)$. The advantage of using the reference domain $\hat{\Omega}$ is that finite element approximations can be performed with respect to that fixed domain without being forced to remesh for every new set of the design variables. The finite element discretization of (1a)-(1b) with respect to a simplicial triangulation $\mathcal{T}_{h}(\Omega)$ of the computational domain $\Omega$ leads to a finite dimensional optimization problem

$$
\begin{gathered}
\inf _{u_{h}, \alpha} J_{h}\left(u_{h}, \alpha\right), \\
\text { subject to } L_{h} u_{h}=b_{h}, h(\alpha) \geq 0,
\end{gathered}
$$

where $u_{h} \in \mathbb{R}^{n}$ is the finite element approximation of the state $u, J_{h}\left(u_{h}, \alpha\right)$ the discretized objective functional and $L_{h} u_{h}=b_{h}$ the algebraic system arising from the finite element discretization of the PDE.

The inequality constrained nonlinear programming problem (2a)-(2b) will be numerically solved by adaptive multilevel path-following primal-dual interiorpoint methods as described in the following subsections. For ease of notation, in the sequel we will drop the subindex $h$.

\section{Adaptive multilevel primal-dual interior point methods}

We couple the inequality constraints in (1b) by logarithmic barrier functions with a barrier parameter $\beta=1 / \mu>0, \mu \rightarrow \infty$, and the equality constraint by a Lagrange multiplier $\lambda \in \mathbb{R}^{n}$. This leads to the saddle point problem

$$
\inf _{u, \alpha} \sup _{\lambda} \mathcal{L}^{(\mu)}(u, \lambda, \alpha),
$$

where $\mathcal{L}^{(\mu)}$ stands for the Lagrangian

$$
\mathcal{L}^{(\mu)}(u, \lambda, \alpha)=B^{(\mu)}(u, \alpha)+\langle\lambda, L u-b\rangle .
$$


Here, $B^{(\mu)}(u, \alpha)$ is the so-called barrier function as given by

$$
B^{(\mu)}(u, \alpha):=J(u, \alpha)-\frac{1}{\mu} \ln (h(\alpha)) .
$$

and $\langle\cdot, \cdot\rangle$ stands for the Euclidean inner product on $\mathbb{R}^{n}$ (for details cf., e.g., Wright (1992)). The central path $\mu \longmapsto x(\mu):=(u(\mu), \lambda(\mu), \alpha(\mu))^{T}$ is given as the solution of the nonlinear system

$$
F(x(\mu), \mu)=\left(\begin{array}{c}
\mathcal{L}_{u}^{(\mu)}(u, \lambda, \alpha) \\
\mathcal{L}_{\lambda}^{(\mu)}(u, \lambda, \alpha) \\
\mathcal{L}_{\alpha}^{(\mu)}(u, \lambda, \alpha)
\end{array}\right)=0
$$

where the subindices refer to the derivatives of the Lagrangian with respect to the primal, the dual, and the design variables. The choice of the barrier parameter strongly influences the performance of the interior-point method. There are static strategies with the Fiacco-McCormick approach as the most prominent one (cf. Fiacco and McCormick (1990)), where the barrier parameter is fixed until an approximate solution has been obtained, and there is a variety of dynamic update strategies (cf. Armand et al. (2007); El-Bakry et al. (1996); Gay et al. (1998); Nocedal et al. (2006); Tits et al. (2003); Ulbrich et al. (2004); Vanderbei and Shanno (1999)). Convergence properties of the Fiacco-McCormick approach have been studied in Byrd et al. (2000) and Wächter and Biegler (2005), whereas a convergence analysis of dynamic update strategies has been addressed in Armand et al. (2007); El-Bakry et al. (1996); Nocedal et al. (2006); Ulbrich et al. (2004).

We consider the solution of (5) by an adaptive continuation method based on the affine invariant convergence theory of Newton-type methods (see, e.g., Deuflhard (2004)).

The adaptive continuation method is a predictor-corrector method with an adaptively determined continuation step size in the predictor and Newton's method as a corrector. It relies on the affine invariant convergence theory of Newton and Newton-type methods and ensures that the iterates stay within a neighborhood (contraction tube) of the central path so that convergence to a local minimum of the original minimization problem can be achieved (cf. Fig. 1)

Predictor Step: The predictor step relies on tangent continuation along the trajectory of the Davidenko equation

$$
F_{x}(x(\mu), \mu) x^{\prime}(\mu)=-F_{\mu}(x(\mu), \mu)
$$

and amounts to the implementation of an explicit Euler step: Given some approximation $\tilde{x}\left(\mu_{k}\right)$ at $\mu_{k}>0$, compute $\tilde{x}^{\left(j_{0}\right)}\left(\mu_{k+1}\right)$, where $\mu_{k+1}=\mu_{k}+$ $\Delta \mu_{k}^{(j)}$, according to 


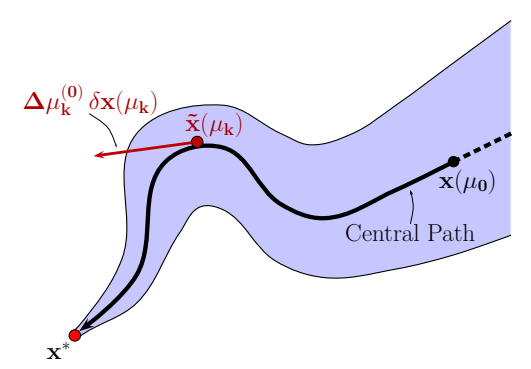

Fig. 1. Predictor step of the adaptive continuation method.

$$
\begin{aligned}
& F_{x}\left(\tilde{x}\left(\mu_{k}\right), \mu_{k}\right) \delta x\left(\mu_{k}\right)=-F_{\mu}\left(\tilde{x}\left(\mu_{k}\right), \mu_{k}\right), \\
& \tilde{x}^{\left(j_{0}\right)}\left(\mu_{k+1}\right)=\tilde{x}\left(\mu_{k}\right)+\Delta \mu_{k}^{(j)} \delta x\left(\mu_{k}\right),
\end{aligned}
$$

starting with $j=0$ ( $j \geq 1$ only if required by the correction step (see below)). We use $\Delta \mu_{0}^{(0)}=\Delta \mu_{0}$ for some given initial step size $\Delta \mu_{0}$, whereas for $k \geq 1$ the predicted step size $\Delta \mu_{k}^{(0)}$ is chosen by

$$
\Delta \mu_{k}^{(0)}:=\left(\frac{\left\|\Delta x^{\left(j_{0}\right)}\left(\mu_{k}\right)\right\|}{\left\|\tilde{x}\left(\mu_{k}\right)-\tilde{x}^{\left(j_{0}\right)}\left(\mu_{k}\right)\right\|} \frac{\sqrt{2}-1}{2 \Theta\left(\mu_{k}\right)}\right)^{1 / 2} \Delta \mu_{k-1},
$$

where $\Delta \mu_{k-1}$ is the computed continuation step size, $\Delta x^{\left(j_{0}\right)}\left(\mu_{k}\right)$ is the first Newton correction (see below), and $\Theta\left(\mu_{k}\right)<1$ is the contraction factor associated with a successful previous continuation step.

Corrector step: As a corrector, we use Newton's method applied to

$$
F\left(x\left(\mu_{k+1}\right), \mu_{k+1}\right)=0
$$

with $\tilde{x}^{\left(j_{0}\right)}\left(\mu_{k+1}\right)$ from $(8 \mathrm{~b})$ as a start vector. In particular, for $\ell \geq 0$ (Newton iteration index) and $j_{\ell} \geq 0$ ( $j$ being the steplength correction index) we compute $\Delta \mathbf{x}^{(j \ell)}\left(\mu_{k+1}\right)$ according to

$$
F_{x}\left(\tilde{x}^{\left(j_{\ell}\right)}\left(\mu_{k+1}\right), \mu_{k+1}\right) \Delta x^{\left(j_{\ell}\right)}\left(\mu_{k+1}\right)=-F\left(\tilde{x}^{\left(j_{\ell}\right)}\left(\mu_{k+1}\right), \mu_{k+1}\right),
$$

update $\tilde{x}^{\left(j_{\ell+1}\right)}\left(\mu_{k+1}\right):=\tilde{x}^{\left(j_{\ell}\right)}\left(\mu_{k+1}\right)+\Delta x^{\left({ }_{\ell}\right)}\left(\mu_{k+1}\right)$ and compute $\overline{\Delta x}^{\left(j_{\ell}\right)}\left(\mu_{k+1}\right)$ as the associated simplified Newton correction

$$
F_{x}\left(\tilde{x}^{\left(j_{\ell}\right)}\left(\mu_{k+1}\right), \mu_{k+1}\right) \overline{\Delta x}^{\left(j_{\ell}\right)}\left(\mu_{k+1}\right)=-F\left(\tilde{x}^{\left(j_{\ell}\right)}\left(\mu_{k+1}\right)+\Delta x^{\left(j_{\ell}\right)}\left(\mu_{k+1}\right), \mu_{k+1}\right) .
$$

We monitor convergence of Newton's method by means of

$$
\Theta^{\left(j_{\ell}\right)}\left(\mu_{k+1}\right):=\left\|\overline{\Delta x}^{\left(j_{\ell}\right)}\left(\mu_{k+1}\right)\right\| /\left\|\Delta x^{\left(j_{\ell}\right)}\left(\mu_{k+1}\right)\right\| .
$$

In case of successful convergence, we set $\tilde{x}\left(\mu_{k+1}\right):=\tilde{x}^{\left(j_{\ell}\right)}\left(\mu_{k+1}\right)$ with $\ell$ being the current Newton iteration index, accept the current step size $\Delta \mu_{k}:=\Delta \mu_{k}^{(j)}$ 


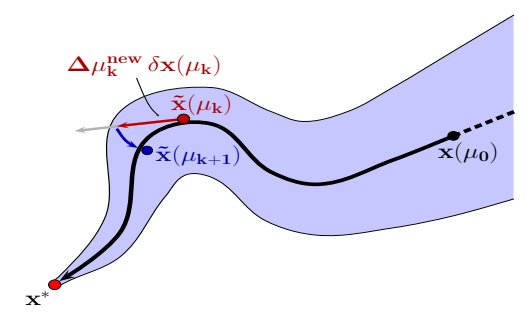

Fig. 2. Correction step of the adaptive continuation method.

with current steplength correction index $j$ and proceed with the next continuation step. However, if the monotonicity test

$$
\Theta^{\left(j_{\ell}\right)}\left(\mu_{k+1}\right)<1
$$

fails for some $j_{\ell} \geq 0$, the predicted steplength $\Delta \mu_{k}^{(j)}$ has been chosen too large so that the predicted solution $\tilde{\mathbf{x}}^{\left(j_{0}\right)}\left(\mu_{k+1}\right)$ is not situated within the Kantorovich neighborhood of $x\left(\mu_{k+1}\right)$, i.e., it is outside the contraction tube around the central path (cf. Fig. 2). The corrector step provides a correction of the steplength for the tangent direction $\delta x\left(\mu_{k}\right)$ such that the new iterate stays within the contraction tube. To do so, the continuation step from (8b) has to be repeated with the reduced step size

$$
\begin{aligned}
\Delta \mu_{k}^{(j+1)} & :=\left(\frac{\sqrt{2}-1}{g\left(\Theta^{(j \ell}\right)}\right)^{1 / 2} \Delta \mu_{k}^{(j)}, \\
g(\Theta) & :=\sqrt{\Theta+1}-1
\end{aligned}
$$

until we either achieve convergence or for some prespecified lower bound $\Delta \mu_{\min }$ observe

$$
\Delta \mu_{k}^{(j+1)}<\Delta \mu_{\min } .
$$

In the latter case, we stop the algorithm and report convergence failure.

The Newton steps are realized by an inexact Newton method featuring righttransforming iterations (cf., e.g., Hoppe et al. (2006); Hoppe and Petrova (2004)). The derivatives occurring in the KKT conditions and the Hessians are computed by automatic differentiation (cf., e.g., Griewank (2000)).

We perform the predictor-corrector scheme in a multilevel framework with respect to a hierarchy of discretizations. We describe the multilevel approach in case of a two-level scheme with the levels $\ell-1$ and $\ell$ (cf. Fig. 3). The prediction is done by nested iteration in such a way that some adaptive continuation steps are performed on the coarser level $\ell-1$ before a predicted value is computed on the finer level $\ell$. The corrector is a Newton multigrid method incorporating a two-level PDE solver featuring appropriate smoothers. The iterates are checked for acceptance by the level $\ell$ monotonicity test. In some 
more detail, we illustrate the two-level scheme in case of two continuation steps on level $\ell-1$. We assume that approximations $x^{\ell-1}\left(\mu_{k}\right)$ and $x^{\ell}\left(\mu_{k}\right)$ are available for some continuation parameter $\mu_{k}$. Firstly, we perform 2 continuation steps with an adaptive choice of the continuation steplengths. Secondly, we use the the level $\ell-1$ approximations $x^{\ell-1}\left(\mu_{k}\right)$ and $x^{\ell-1}\left(\mu_{k+2}\right)$ as well as the level 1 approximation $x^{\ell}\left(\mu_{k}\right)$ to obtain a level 1 prediction at $\mu_{k+2}$. This approximation is then corrected by the two-level Newton multigrid scheme and checked for acceptance by the level $\ell$ monotonicity test. In the general case of more than 2 levels, the multilevel predictor-corrector continuation method consists of a recursive application of the two-level scheme.

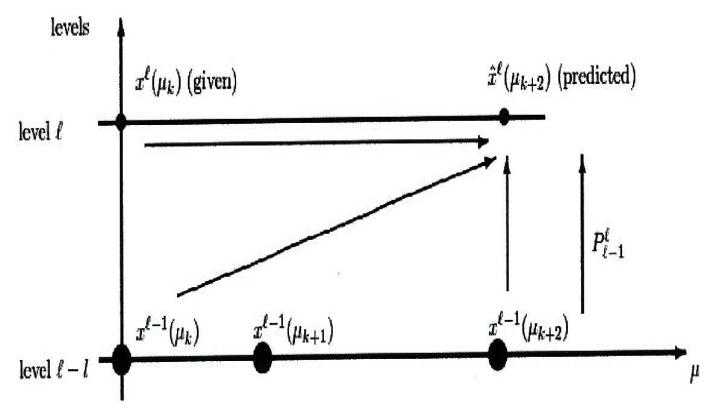

Fig. 3. Two-level predictor-corrector scheme

\section{Numerical results}

Microfluidic biochips are used in pharmaceutical, medical and forensic applications as well as in academic research and development for high throughput screening, genotyping and sequencing by hybridization in genomics, protein profiling in proteomics, and cytometry in cell analysis (cf., e.g., Pollard and Castrodale (2003); Wagner et al. (2002)). Recent nanotechnological devices are surface acoustic wave driven biochips with integrated fluidics on top of the chip consisting of a lithographically produced network of channels and reservoirs (see Fig. 4 (left)). The core of the technology are nanopumps featuring surface acoustic waves generated by electric pulses of high frequency. These waves propagate like a miniaturized earthquake, enter the fluid filled channels and thus cause a flow which transports the DNA or protein containing liquid along the network to a reservoir where the chemical analysis is performed (see, e.g., Wixforth et al. (2002, 2004). Between the channels and the reservoirs are capillary barriers (cf. Fig. 4 (right)) which have to be designed in such a way that a precise filling of the reservoirs is guaranteed. 

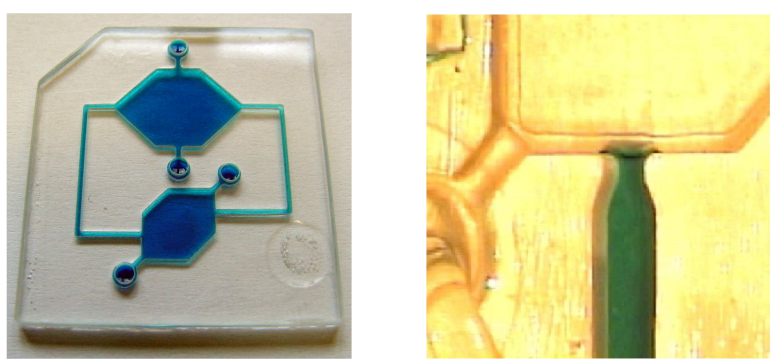

Fig. 4. Microfluidic biochip (left) and capillary barrier (right)

Mathematical models for SAW biochips are based on the linearized equations of piezoelectricity in $Q_{1}:=\left(0, T_{1}\right) \times \Omega_{1}$

$$
\begin{aligned}
\rho_{1} \frac{\partial^{2} u_{i}}{\partial t^{2}}-\frac{\partial}{\partial x_{j}} c_{i j k l} \frac{\partial u_{k}}{\partial x_{l}}-\frac{\partial}{\partial x_{j}} e_{k i j} \frac{\partial \Phi}{\partial x_{k}} & =0 \\
\frac{\partial}{\partial x_{j}} e_{j k l} \frac{\partial u_{k}}{\partial x_{l}}-\frac{\partial}{\partial x_{j}} \epsilon_{j k} \frac{\partial \Phi}{\partial x_{k}} & =0
\end{aligned}
$$

with appropriate initial conditions at $t=0$ and boundary conditions on $\Gamma_{1}:=$ $\partial \Omega_{1}$. Here, $\rho_{1}$ and $\mathbf{u}=\left(u_{1}, u_{2}, u_{3}\right)^{T}$ denote the density of the piezoelectric material and the mechanical displacement vector. Moreover, $\boldsymbol{\epsilon}=\left(\epsilon_{i j}\right)$ stands for the permittivity tensor and $\Phi$ for the electric potential. The tensors $\mathbf{c}=$ $\left(c_{i j k l}\right)$ and $\mathbf{e}=\left(e_{i k l}\right)$ refer to the forth order elasticity tensor and third-order piezoelectric tensor, respectively.

The modeling of the micro-fluidic flow is based on the compressible NavierStokes equations in $Q_{2}:=\left(0, T_{2}\right) \times \Omega_{2}$

$$
\begin{aligned}
\rho_{2}\left(\frac{\partial \mathbf{v}}{\partial t}+(\mathbf{v} \cdot \nabla) \mathbf{v}\right) & =-\nabla p+\eta \Delta \mathbf{v}+\left(\zeta+\frac{\eta}{3}\right) \nabla(\nabla \cdot \mathbf{v}) \\
\frac{\partial \rho_{2}}{\partial t}+\nabla \cdot\left(\rho_{2} \mathbf{v}\right) & =0 \\
\mathbf{v}(x+\mathbf{u}(x, t), t) & =\frac{\partial \mathbf{u}}{\partial t}(x, t) \quad \text { on }\left(0, T_{2}\right) \times \Gamma_{2}
\end{aligned}
$$

with suitable initial conditions at $t=0$. Here, $\rho_{2}, \mathbf{v}=\left(v_{1}, v_{2}, v_{3}\right)^{T}$ and $p$ are the density of the fluid, the velocity, and the pressure. $\eta$ and $\zeta$ refer to the shear and the bulk viscosity. The boundary conditions include the time derivative $\partial \mathbf{u} / \partial t$ of the displacement of the walls $\Gamma_{2}=\partial \Omega_{2}$ of the microchannels caused by the surface acoustic waves. The induced fluid flow involves extremely different time scales. The damping of the jets created by the SAWs happens on a time scale of nanoseconds, whereas the resulting acoustic streaming reaches an equilibrium on a time scale of milliseconds. We perform a separation of the time-scales by homogenization using an expansion $\mathbf{v}=\mathbf{v}_{0}+\varepsilon \mathbf{v}^{\prime}+\varepsilon^{2} \mathbf{v}^{\prime \prime}+O\left(\varepsilon^{3}\right)$ of the velocity $\mathbf{v}$ in a scale parameter $\varepsilon>0$ 
representing the maximal displacement of the walls and analogous expansions of the pressure $p$ and the density $\rho_{2}$. We set $\mathbf{v}_{1}:=\varepsilon \mathbf{v}^{\prime}, \mathbf{v}_{2}:=\varepsilon^{2} \mathbf{v}^{\prime \prime}$ and define $p_{i}, \rho_{2, i}, 1 \leq i \leq 2$, analogously. Time-averaging the second order (in $\varepsilon$ ) system according to $\langle w\rangle:=T^{-1} \int_{t_{0}}^{t_{0}+T} w d t, T:=2 \pi / \omega$, we arrive at the following Stokes equations in $\Omega_{2}$

$$
\begin{aligned}
-\eta \Delta \mathbf{v}_{2}-\left(\zeta+\frac{\eta}{3}\right) \nabla\left(\nabla \cdot \mathbf{v}_{2}\right)+\nabla p_{2} & =\left\langle-\rho_{2,1} \frac{\partial \mathbf{v}_{1}}{\partial t}-\rho_{2,0}\left[\nabla \mathbf{v}_{1}\right] \mathbf{v}_{1}\right\rangle \\
\rho_{2,0} \nabla \cdot \mathbf{v}_{2} & =\left\langle-\nabla \cdot\left(\rho_{2,1} \mathbf{v}_{1}\right)\right\rangle \\
\mathbf{v}_{2} & =-\left\langle\left[\nabla \mathbf{v}_{1}\right] \mathbf{u}\right\rangle \text { on } \Gamma_{2} .
\end{aligned}
$$

which describe the stationary flow pattern, called acoustic streaming, resulting after the relaxation of the high frequency surface acoustic waves (for further details we refer to Antil et al. (2008); Gantner et al. (2007); Köster (2007)).

Table 1. History of the adaptive multilevel predictor-corrector strategy (Capillary barriers, 4 Levels)

\begin{tabular}{|c|c|c|c|c|}
\hline level & $k$ & $\mu$ & $\Delta \mu$ & $\Delta J$ \\
\hline 1 & 0 & $2.0 \mathrm{E}+02$ & & \\
& & & & $2.83 \mathrm{E}+00$ \\
& 1 & $6.3 \mathrm{E}+02$ & $4.3 \mathrm{E}+02$ & $1.87 \mathrm{E}-05$ \\
& 2 & $1.1 \mathrm{E}+03$ & $4.9 \mathrm{E}+02$ & $3.40 \mathrm{E}-06$ \\
& 3 & $1.6 \mathrm{E}+03$ & $5.1 \mathrm{E}+02$ & $1.09 \mathrm{E}-06$ \\
& 4 & $2.3 \mathrm{E}+03$ & $6.8 \mathrm{E}+02$ & $5.70 \mathrm{E}-07$ \\
& 5 & $3.5 \mathrm{E}+03$ & $1.1 \mathrm{E}+03$ & $3.63 \mathrm{E}-07$ \\
& 6 & $5.3 \mathrm{E}+03$ & $1.9 \mathrm{E}+03$ & $1.99 \mathrm{E}-07$ \\
& 7 & $8.8 \mathrm{E}+03$ & $3.5 \mathrm{E}+03$ & $1.02 \mathrm{E}-07$ \\
& 8 & $1.6 \mathrm{E}+04$ & $7.3 \mathrm{E}+03$ & $4.50 \mathrm{E}-08$ \\
\hline 2 & 2 & $1.1 \mathrm{E}+03$ & $9.2 \mathrm{E}+02$ & \\
& 4 & $2.3 \mathrm{E}+03$ & $1.2 \mathrm{E}+03$ & \\
& 6 & $5.3 \mathrm{E}+03$ & $3.0 \mathrm{E}+03$ & \\
& 8 & $1.6 \mathrm{E}+04$ & $1.1 \mathrm{E}+04$ & \\
\hline 3 & 4 & $2.3 \mathrm{E}+03$ & $2.1 \mathrm{E}+03$ & \\
& 8 & $1.6 \mathrm{E}+04$ & $1.4 \mathrm{E}+04$ & \\
4 & 8 & $1.6 \mathrm{E}+04$ & $1.6 \mathrm{E}+04$ & \\
\hline
\end{tabular}

We have considered the optimal design of a capillary barrier for a domain consisting of part of a microchannel close to a reservoir with two passive outlet valves to allow for an outflow in case of the stopping mode of the barrier (cf. Fig. 5). The objective functional $J$ has been chosen of tracking type according to

$$
J\left(\mathbf{v}_{2}, p_{2}, \alpha\right):=\frac{1}{2} \int_{\Omega(\alpha)}\left|\mathbf{v}_{2}-\mathbf{v}_{2}^{d}\right|^{2} d x+\frac{1}{2} \int_{\Omega(\alpha)}\left|p_{2}-p_{2}^{d}\right|^{2} d x
$$



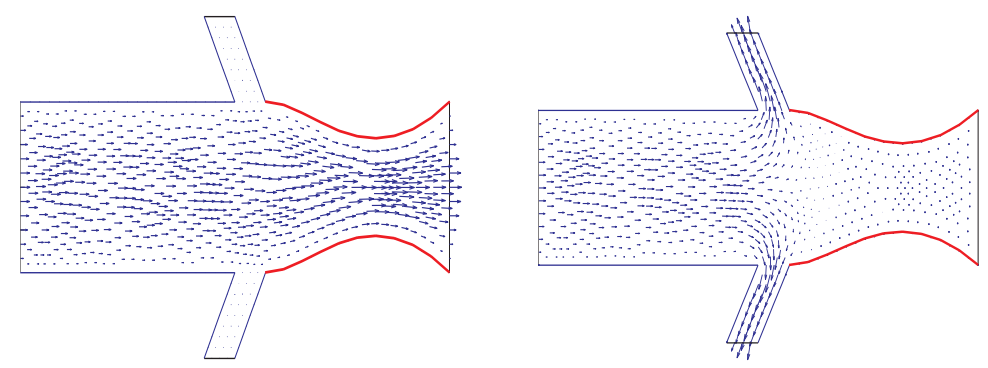

Fig. 5. Optimally designed capillary barrier:Velocity profile in the flow mode (left) and in the stopping mode (right)

subject to the Stokes system (15a)-(15c) with Signorini type boundary conditions at the junction between the microchannel and the reservoir. We have used $m=16$ Bézier control points of a Bézier curve representation of the barrier as design variables subject to bilateral constraints. Table 1 contains the history of the multilevel interior-point method described in the previous section in case of four levels $1 \leq \ell \leq 4$ with 2362 degrees of freedom (DOFs) on the coarsest grid (level 1) and 141634 DOFs on the finest grid (level 4). The number $k$ indicates the continuation steps, $\mu_{k}$ and $\Delta \mu_{k}:=\mu_{k}-\mu_{k-1}$ refer to the inverse of the barrier parameter $\beta_{k}$ and the increment in $\mu_{k}$, and $\Delta J_{k}$ is the difference between the corresponding values of the objective functional. We have performed two continuation steps on a coarser grid before proceeding by nested iteration to the next finer grid, and we have used $\left|\triangle J_{k}\right|<T O L$ with $T O L:=1.0 E-07$ as a termination criterion for the continuation process. Fig. 5 displays the optimal design of the barrier and the associated velocity profiles in the flow mode (fluid flow into the reservoir) and in the stopping mode (backflow). For further results and a comparison with other continuation methods and update strategies of the barrier parameter we refer to Antil, Hoppe and Linsenmann (2008).

Acknowledgements. The second and third authors acknowledge support by the German National Science Foundation within the Priority program SPP 1253 'Optimization with Partial Differential Equations'. The work of the authors has been further supported by the NSF under Grant-No. DMS-0511611, DMS-0707602.

\section{References}

G. Allaire; Shape Optimization by the Homogenization Method. Springer, Berlin-Heidelberg-New York, 2002. 
H. Antil, A. Gantner, R.H.W. Hoppe, D. Köster, K.G. Siebert, and A. Wixforth; Modeling and Simulation of Piezoelectrically Agitated Acoustic Streaming on Microfluidic Biochips. In: Proc. 17th Int. Conf. on Domain Decomposition Methods (Langer, U. et al.; eds.), Lecture Notes in Computational Science and Engineering, Vol. 60, Springer; Berlin Heidelberg-New York, 2008.

H. Antil, R.H.W. Hoppe, and C. Linsenmann; Optimal design of stationary flow problems by path-following interior-point methods. submitted

P. Armand, J. Benoist, and D. Orban; Dynamic updates of the barrier parameter in primal-dual methods for nonlinear programming. to appear in Computational Optimization and Applications, 2007.

M.P. Bendsøe; Optimization of Structural Topology, Shape, and Material. Springer, Berlin-Heidelberg-New York, 1995.

R.H. Byrd, J.C. Gilbert, and J. Nocedal; A trust region method based on interior point techniques for nonlinear programming. Math. Programming 89, 149-185, 2000.

R.H. Byrd, M.E. Hribar, and J. Nocedal; An interior point algorithm for large scale nonlinear programming. SIAM J. Optimization 9, 877-900, 1999.

M.C. Delfour and J.P. Zolesio; Shapes and Geometries: Analysis, Differential Calculus and Optimization. SIAM, Philadelphia, 2001.

P. Deuflhard; Newton Methods for Nonlinear Problems. Affine Invariance and Adaptive Algorithms. Springer, Berlin-Heidleberg-New York, 2004.

A.S. El-Bakry, R.A. Tapia, T. Tsuchiya, and Y. Zhang; On the formulation and theory of the Newton interior-point method for nonlinear programming. Journal of Optimization Theory and Applications 89, 507-541, 1996.

K. Eppler and H. Harbrecht; Second order shape optimization using wavelet BEM. Optim. Methods Software 21, 135-153, 2006.

A.V. Fiacco and G.P. McCormick; Nonlinear Programming: Sequential Unconstrained Minimization Techniques. SIAM, Philadelphia, 1990.

A. Forsgren, Ph.E. Gill, and M.H. Wright; Interior methods for nonlinear optimization. SIAM Rev. 44, 522-597, 2002.

A. Gantner, R.H.W. Hoppe, D. Köster, K.G. Siebert, and A. Wixforth; Numerical simulation of piezoelectrically agitated surface acoustic waves on microfluidic biochips. Comp. Visual. Sci. 10, 145-161, 2007.

M.D. Gay, M.L. Overton, and M.H. Wright; A primal-dual interior method for nonconvex nonlinear programming. In: Advances in Nonlinear Programming (Yuan Y, ed.), pp. 31-56, Kluwer, Dordrecht, 1998.

A. Griewank; Evaluating Derivatives, Principles and Techniques of Automatic Differentiation. SIAM, Phildelphia, 2000.

J. Haslinger and P. Neittaanmäki; Finite Element Approximation for Optimal Shape Design: Theory and Applications. John Wiley \& Sons, Chichester, 1988.

J. Haslinger and R.A.E. Mäkinen; Introduction to Shape Optimization: Theory, Approximation, and Computation. SIAM, Philadelphia, 2004. 
R.H.W. Hoppe, C. Linsenmann, and S.I. Petrova; Primal-dual Newton methods in structural optimization. Comp. Visual. Sci. 9, 71-87, 2006.

R.H.W. Hoppe and S.I. Petrova; Primal-dual Newton interior-point methods in shape and topology optimization. Numer. Linear Algebra Appl. 11, 413429, 2004.

R.H.W. Hoppe, S.I. Petrova, and V. Schulz; A primal-dual Newton-type interior-point method for topology optimization. Journal of Optimization: Theory and Applications 114, 545-571, 2002.

D. Köster; Numerical simulation of acoustic streaming on SAW-driven biochips. SIAM J. Comp. Sci. 29, 2352-2380, 2007.

B. Mohammadi and O. Pironneau; Applied Shape Optimization for Fluids. Oxford University Press, Oxford, 2001.

J. Nocedal, A. Wächter, and R.A. Waltz; Adaptive barrier update strategies for nonlinear interior methods. Research Report RC 23563, IBM T. J. Watson Research Center, Yorktown, 2006.

O. Pironneau; Optimal Shape Design for Elliptic Systems. Springer, BerlinHeidelberg-New York, 1984.

J. Pollard and B. Castrodale; Outlook for DNA microarrays: emerging applications and insights on optimizing microarray studies. Report. Cambridge Health Institute, Cambridge, 2003.

J. Sokolowski and J.P. Zolesio; Introduction to Shape Optimization. Springer, Berlin-Heidelberg-New York, 1992.

A.L. Tits, A. Wächter, S. Bakhtiari, T.J. Urban, and C.T. Lawrence; $A$ primal-dual interior-point method for nonlinear programming with strong global and local convergence properties. SIAM J. on Optimization 14, 173199, 2003.

M. Ulbrich, S. Ulbrich, and L. Vicente; A globally convergent primal-dual interior point filter method for nonconvex nonlinear programming. Math. Programming 100, 379-410, 2004.

R.J. Vanderbei and D.F. Shanno; An interior point algorithm for nonconvex nonlinear programming. Computational Optimization and Applications 13, 231-252, 1999.

A. Wächter and L.T. Biegler; Line search filter methods for nonlinear programming: motivation and global convergence. SIAM J. on Optimization 16, 1-31, 2005.

P. Wagner, M.X. Tan, F.G. Zaugg, and P.F. Indermühle; Protein biochips: protein analysis on a small scale. mst news 5, 44, 2002.

A. Wixforth, J. Scriba, and G. Gauer; Flatland fluidics. mst news 5, 42-43, 2002.

A. Wixforth, C. Strobl, C. Gauer, A. Toegl, J. Scriba, T. Guttenberg; Acoustic manipulation of small droplets. Anal. Bioanal. Chem. 379, 982-991.

M.H. Wright; Interior methods for constrained optimization. Acta Numerica 1, 341-407,1992. 